\title{
Fontes alternativas de cálcio e fósforo para poedeiras comerciais
}

\section{Humberto Pena Couto ${ }^{1}$, Víctor Libardo Hurtado Nery ${ }^{2}$, José Brandão Fonseca ${ }^{1}$, Julien Chiquieri $^{3}$, Laura Chaffin Rocha Carneiro ${ }^{4}$, Cláudio Teixeira Lombardi ${ }^{5}$}

\author{
1 UENF/CCTA/LZNA, Av. Alberto Lamego, 2000, Campos dos Goytacazes, RJ, CEP: 28013-602. \\ 2 Universidad de los Llanos - Colombia. \\ ${ }^{3}$ Doutorando em Produção Animal - UENF. \\ ${ }^{4}$ Curso de Graduação em Zootecnia - UENF. \\ 5 Técnico superior do Laboratório de Análises de Alimentos - UENF.
}

RESUMO - Este estudo foi realizado com o objetivo de avaliar o fosfato monoamônio (MAP) como fonte de fósforo e o calcário calcítico Paraíso (CP) com mais alto nível de magnésio (1,92\%) como fonte de cálcio para poedeiras comerciais. Foram alojadas em gaiolas 280 poedeiras brancas Dekalb durante o período de 46 a 58 semanas de idade. O delineamento experimental foi o inteiramente casualizado com quatro tratamentos (fontes de cálcio e fósforo), sete repetições e dez aves por unidade experimental. As fontes de cálcio e fósforo avaliadas foram: FBC/CB - fosfato bicálcico e calcário calcítico Barroso (cinza); FBC/CP - fosfato bicálcico e calcário calcítico Paraíso (branco); MAP/CB - fosfato monoamônio e calcário calcítico Barroso; MAP/CP - fosfato monoamônio e calcário calcítico Paraíso. Foram avaliados a produção, o peso médio e a massa dos ovos, o consumo de ração, a conversão alimentar e o percentual e a espessura da casca. A produção, o peso médio e a massa de ovos melhoraram com a utilização de fosfato monoamônio e ambas as fontes de calcário avaliadas. O consumo de ração aumentou e a conversão alimentar melhorou com a utilização de MAP como fonte de fósforo. O percentual e a espessura de casca dos ovos não foram significativamente influenciados pela fonte de cálcio da dieta, logo, o nível maior de magnésio e a menor granulometria do calcário Paraíso não comprometeram a qualidade da casca dos ovos. Não foram observadas alterações na consistência das fezes e na taxa de mortalidade das poedeiras em produção. A excreção de nitrogênio foi $10 \%$ maior nas aves que consumiram o fosfato monoamônio como fonte de fósforo. O fosfato monoamônio pode ser utilizado como fonte alternativa de fósforo em substituição ao fosfato bicálcico. A utilização de calcário Paraíso é tecnicamente viável na alimentação de poedeiras comerciais e não prejudica a produção e a qualidade da casca dos ovos.

Palavras-chave: calcário, fosfato monoamônio, qualidade da casca, produção de ovos

\section{Alternative sources of calcium and phosphorus for commercial laying hens}

\begin{abstract}
The objective of this study was to evaluate the monoammonium phosphate (MAP) as a source of phosphorus and Paraíso limestone (CP) with higher magnesium levels (1.92\%) as a source of calcium for commercial layers. A total of 280 white Dekalb chickens were housed in individual cages during the period from 46 to 58 weeks old. A completely randomized experimental design, with four diets, seven replicates and ten birds per experimental unit were used. The diets were: FBC/CB - dicalcium phosphate and Barroso limestone (gray); FBC/CP - dicalcium phosphate and Paraíso limestone (white); MAP/CB - monoammonium phosphate and Barroso limestone; MAP/CP - monoammonium phosphate and Paraíso limestone. Egg production, mean weight and egg mass; feed intake and feed conversion were evaluate, and also shell percentage and thickness. Egg production, mean egg weight and mass improved with the use MAP with both evaluated limestone. Feed intake and feed conversion improved with utilization of MAP as source of phosphorus. Egg shell percentage and thickness were not affected by calcium source in the diet, hence, the higher level of $\mathrm{Mg}$ and finer particles of Paraíso limestone did not impaired egg shell quality. No alterations were observed in feces consistency and survival rate of the layers. The nitrogen excretion was $10 \%$ higher for birds that received MAP as a phosphorus source. Monoammonium phosphate could be used as an alternative source of phosphorus replacing dicalcium phosphate; and Paraíso limestone is technically viable for feeding commercial layers without problems for egg production and shell quality.
\end{abstract}

Key Words: egg production, limestone, monoammonium phosphate, shell quality 


\section{Introdução}

A produção de ovos comerciais no Brasil é uma atividade de grande importância sócio-econômica. Em 2006, considerando apenas as granjas comerciais brasileiras, foram produzidas 20 a 22 bilhões de unidades.

Existe grande variação na qualidade dos fosfatos utilizados na nutrição animal. Essa variação pode ser explicada pelos processos de produção, pelas concentrações de fósforo (P) e pela composição química dos fosfatos (PCS Fosfatos do Brasil, 2003). O fosfato bicálcico é uma fonte mineral muito utilizada na nutrição de aves e com bastante padronização. O fosfato monoamônio, também conhecido como MAP, é obtido pelo tratamento do ácido fosfórico com hidróxido de amônio desfluorizado após sua extração dos fosfatos de rocha (Veloso,1991).

Segundo Butolo (2002), para que seja utilizado em suplementos minerais e rações, o fósforo tem que se apresentar em forma química adequada, ou seja, com alta biodisponibilidade. Contudo, comprovada sua viabilidade zootécnica, a opção entre as diferentes fontes é realizada com base no custo por unidade de fósforo biodisponível, e não na unidade de fósforo total. $\mathrm{O}$ fosfato monoamônio tem aproximadamente $24 \%$ de fósforo total, 0,30\% de flúor e $11 \%$ de nitrogênio nãoprotéico. A disponibilidade de fósforo desse fosfato em relação ao bicálcico é de 95 a 100\% (Butolo, 2002), no entanto, as Tabelas brasileiras para aves e suínos (2005) reportam valor relativo mais elevado, de $108 \%$.

O calcário é uma rocha sedimentar de origem orgânica composta principalmente de carbonato de cálcio na forma dos minerais calcita e aragonita. Em estado puro, o calcário pode ser branco, cinzento, creme ou amarelo (HM Minérios Industriais, 1999). De acordo com Axe (1989), a solubilidade in vitro dos calcários, e não sua simples granulometria, é o melhor preditor da resposta de aves poedeiras. Zhang \& Coon (1997) observaram aumento de retenção de calcário na moela das aves quando o nível de cálcio da ração foi alto ou a solubilidade in vitro do calcário foi baixa, além de menor solubilidade in vivo do cálcio quando aumentaram o nível de cálcio na dieta.

Fassini et al. (2004) relataram que a falta de conhecimento das características fisico-químicas dos calcários pode ocasionar variação das exigências nutricionais obtidas em pesquisas científicas, o que leva muitos nutricionistas à utilização errônea de altos níveis de cálcio em rações para poedeiras comerciais.

Objetivou-se com este trabalho avaliar o fosfato monoamônio como fonte alternativa de fósforo e o calcário calcítico Paraíso com mais alto teor de magnésio como fonte de cálcio para poedeiras comerciais na fase de produção.

\section{Material e Métodos}

O experimento foi realizado no setor de avicultura da Unidade de Apoio à Pesquisa do Laboratório de Zootecnia e Nutrição Animal da Universidade Estadual do Norte Fluminense-UENF, Campos dos Goytacazes, Rio de Janeiro, durante o período de junho a agosto de 2006. Utilizaram-se 280 poedeiras brancas da linhagem Dekalb provenientes da Granja Planalto, de Uberlândia, Minas Gerais, no período de 46 a 58 semanas de idade. As aves foram alojadas individualmente em gaiolas com comedouro tipo calha e bebedouros tipo nipple. O período experimental foi dividido em três subperíodos de quatro semanas. O programa de luz utilizado neste período foi o de 16 horas de luz constante controlada por um timer digital, de modo que a iluminação foi fornecida por lâmpadas fluorescentes de 40 watts.

Avaliaram-se como tratamentos os fosfato bicálcico e calcário calcítico Barroso (FBC/CB); fosfato bicálcico e calcário calcítico Paraíso (FBC/CP); fosfato monoamônio e calcário calcítico Barroso (MAP/CB); fosfato monoamônio e calcário calcítico paraíso (MAP/CP).

As rações experimentais (Tabela 1), todas isonutritivas, continham (por kg) 2.820 kcal de energia metabolizável; 16,5\% de proteína bruta; 0,62\% de metionina+cistina digestível; $0,77 \%$ de lisina digestível; $4,2 \%$ de cálcio e 0,42\% de fósforo disponível.

A composição dos alimentos, exceto a do calcário Paraíso (33,8\% de Ca e 1,92\% de Mg) e do fosfato monoamônio (MAP) (Pec-Fos - Cargill) (25\% de fósforo disponível e 0,35\% de flúor), baseou-se nas recomendações descritas nas tabelas brasileiras de aves e suínos (Rostagno et al., 2005). As exigências nutricionais e as estimativas de consumo de ração das poedeiras foram obtidas no manual da linhagem Dekalb.

O diâmetro geométrico médio (DGM) e o desvio-padrão geométrico (DPG) das partículas dos calcários foram determinados utilizando-se a metodologia tradicional de avaliação da granulometria (Butolo, 2002) com peneiras de 1,$00 ; 0,50 ; 0,25$; e $0,15 \mathrm{~mm}$. As quantidades retidas nas peneiras foram registradas e os valores foram analisados pelo software Gransuave, desenvolvido pela EMBRAPACNPSA, para cálculo do DGM e DPG.

A solubilidade aparente dos calcários foi avaliada segundo procedimentos propostos por Zhang \& Coon (1997), descritos por Butolo (2002). Os calcários foram submetidos à solubilização em solução de $\mathrm{HCl} 0,1 \mathrm{~N}$ por 24 horas e o $\mathrm{pH}$ foi medido em quatro tempos $(0,5 ; 1,0 ; 17,0 \mathrm{e}$ 24,0 horas). Os resultados de solubilidade foram obtidos correlacionando a variação do $\mathrm{pH}$ observado nos tempos e a solubilidade padrão proposta pelos autores. Para melhorar 
Tabela 1 - Composição das rações experimentais

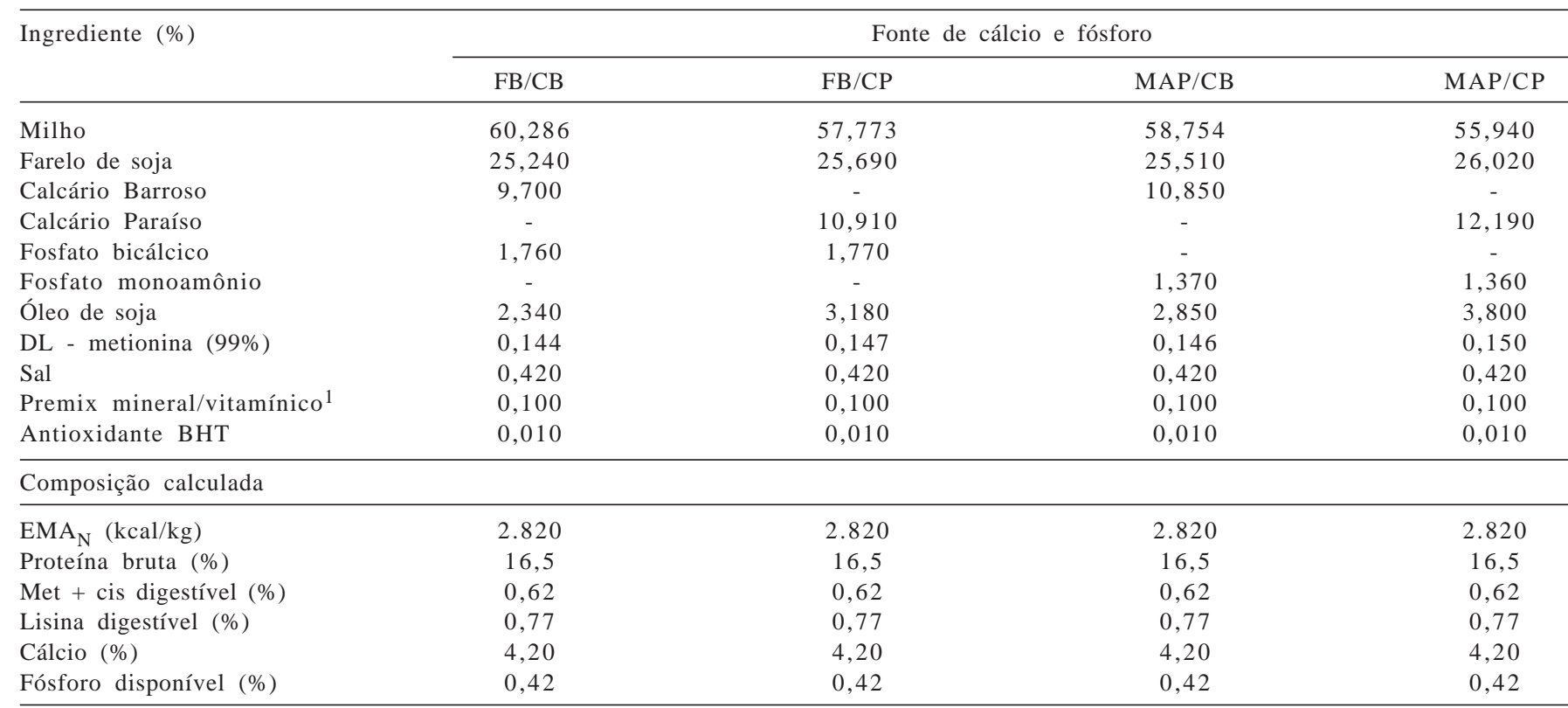

FBC/CB - fosfato bicálcico e calcário calcítico Barroso (cinza); FBC/CP- fosfato bicálcico e calcário calcítico Paraíso (branco); MAP/CB - fosfato monoamônio e calcário calcítico Barroso; MAP/CP - fosfato monoamônio e calcário calcítico Paraíso.

${ }_{1}$ Polimix PR 101 - FATEC (quantidade/kg): vit. A - 8.000.000 UI; vit. $\mathrm{D}_{3}-2.100 .000 \mathrm{UI}$; vit. E - $700 \mathrm{mg}$; vit. $\mathrm{K}-2.000 \mathrm{mg}$; vit. $\mathrm{B}_{1}-1.000 \mathrm{mg}$; vit. $\mathrm{B}_{2}$ -

$3.000 \mathrm{mg}$; vit. $B_{6}-700 \mathrm{mg}$; vit. $B_{12}-6000 \mathrm{mcg}$; ácido nicotínico - $20000 \mathrm{mg}$; ácido fólico - $100 \mathrm{mg}$; pantotenato de cálcio - $10.000 \mathrm{mg}$; biotina - $10 \mathrm{mg}$

$\mathrm{Cu}-6000 \mathrm{mg} ; \mathrm{Zn}-50.000 \mathrm{mg} ; \mathrm{Fe}-50.000 \mathrm{mg}$; I $1.000 \mathrm{mg} ; \mathrm{Mn}-55.000 \mathrm{mg}$; Se - $200 \mathrm{mg}$; antioxidante $-2.000 \mathrm{mg}$.

as estimativas das solubilidades (Y), ajustou-se uma equação quadrática ( $Y=2,0504+180,9464 X-96,0373 X^{2}$, $\left.\mathrm{R}^{2}=99,8\right)$ com os valores obtidos nas variações de $\mathrm{pH}(\mathrm{X})$.

A qualidade dos ovos foi avaliada utilizando-se o peso das cascas após lavagem e secagem em estufa ventilada a $105^{\circ} \mathrm{C}$ por 24 horas e pesagem em balança de precisão (0,01 g). Posteriormente, o percentual da casca foi calculado em relação ao peso do ovo (\%casca = peso casca / peso ovo $\times 100$ ), conforme proposto por Abdallah et al. (1993). A espessura da casca foi avaliada em três pontos eqüidistantes da região equatorial do ovo, utilizando-se um micrômetro externo da marca Mitutoyo, modelo 103-137 (+/- 0,002 mm). A espessura da casca foi obtida pela média de três mensurações eqüidistantes na região equatorial do ovo.

O delineamento experimental foi o inteiramente casualizado com quatro tratamentos (fontes de fósforo e de cálcio), sete repetições e dez aves por unidade experimental. Avaliaram-se a produção de ovos, o peso médio dos ovos, a massa dos ovos (\% de produção de ovos $\times$ peso médio doso ovos/100), o consumo de ração, a conversão alimentar (kg de ração consumida/kg de ovo produzido), a porcentagem de cascas dos ovos e a espessura da casca dos ovos.

Os resultados foram submetidos a análises estatísticas utilizando-se o Sistema de Análises Estatísticas e Genéticas -SAEG, versão 8.0 (UFV, 1999). As médias das características avaliadas foram comparadas pelo teste de Tukey a $5 \%$.

\section{Resultados e Discussão}

Na avaliação da granulometria, os resultados de DGM e DPG obtidos para os calcários Paraíso e Barroso foram: 293 mícrons e 1,64; 405 mícrons e 2,38, respectivamente. O aspecto físico diferiu entre os calcários estudados: o calcário Paraíso tem coloração branca e granulometria mais fina, com diâmetro geométrico médio inferior e tamanho das partículas mais uniforme, em decorrência do menor DPG.

Resultados de pesquisas (Axe, 1989; Rao \& Roland, 1989; Bronner,1993) comprovam a importância da granulometria do calcário na alimentação de poedeiras. A granulometria tem efeito na taxa de solubilização do calcário durante os processos digestivos e sua retenção é mais prolongada na moela, o que beneficia a manutenção dos níveis plasmáticos de cálcio durante o período de formação da casca do ovo. Entretanto, este aspecto não foi observado entre os dois calcários estudados, pois ambos são de granulometria baixa e não comprometem a qualidade da casca dos ovos.

A solubilidade dos calcários diferiu no tempo avaliado (Tabela 2). O calcário Paraíso foi solubilizado em taxas mais elevadas que as do calcário Barroso durante as primeiras horas de consumido. A granulometria mais fina, decorrente do menor diâmetro geométrico médio, contribuiu muito para esta rápida solubilidade inicial. Entretanto, com o passar do tempo, a diferença diminuiu até que, às 24 horas pósingestão, suas solubilidades se tornaram semelhantes e 
Tabela 2 - Solubilidades dos calcários Barroso e Paraíso

\begin{tabular}{lccccc}
\hline \multirow{2}{*}{ Tempo } & \multicolumn{2}{c}{$\Delta \mathrm{pH}$} & & \multicolumn{2}{c}{$\%$ Solubilidade } \\
\cline { 2 - 3 } \cline { 5 - 5 } & Barroso & Paraíso & & Barroso & Paraíso \\
\hline 0,5 & 0,13 & 0,34 & & 24 & 53 \\
1,0 & 0,22 & 0,41 & & 38 & 61 \\
17,0 & 0,34 & 0,49 & & 53 & 68 \\
24,0 & 0,44 & 0,55 & & 64 & 73 \\
\hline
\end{tabular}

tenderam a se igualar nas próximas horas. Esses resultados divergem da afirmativa de Rao \& Roland (1989) de que a solubilidade aumenta quando o calcário é fornecido em partículas maiores. Zhang \& Coon (1997) relataram que ocorre aumento de retenção de calcário na moela das aves quando o nível de cálcio da ração é alto, o que pode explicar a redução da solubilidade do calcário Paraíso em relação ao calcário Barroso no período avaliado.

Segundo Sugahara (1993), o ovo permanece 18 a 20 horas no aparelho reprodutor, no qual ocorre a formação da casca. Como a calcificação ocorre principalmente na segunda metade do tempo de permanência neste órgão (12 a 13 horas), não foi previsto neste estudo comprometimento da formação da casca dos ovos pelos calcários, uma vez que a solubilidade é de 40 a 50\%, logo, os níveis plasmáticos de cálcio foram altos para ambos os calcários estudados neste período.

A produção, o peso médio e a massa de ovos foram $(\mathrm{P}<0,05)$ maiores nas aves que receberam MAP como fonte de fósforo, independentemente da fonte de cálcio utilizada (Tabela 3). Este resultado valida tecnicamente a utilização desta fonte em substituição ao fosfato bicálcico, o que, no entanto, depende da avaliação econômica, que adequadamente deve ser realizada considerando o custo por unidade de fósforo disponível das fontes comparativas em questão.

O fosfato monoamônio, além de fornecer fósforo, também causa ingestão de nitrogênio não-protéico pelas aves, que não contribui para a síntese protéica em animais monogástricos. O nitrogênio ingerido, depois de absorvido, é metabolizado e seu excesso é eliminado via ciclo do ácido úrico, um processo que utiliza energia (ATP). Esse desvio da energia metabolizável pode ter promovido redução da massa de ovos, mas os resultados observados não confirmaram esta premissa.

De acordo com os resultados da análise laboratorial, o nitrogênio excedente foi eliminado pelas excretas, uma vez que os excrementos das aves que receberam o fosfato monoamônio apresentaram, em média, 6,01\% de nitrogênio, enquanto os daquelas que receberam fosfato bicálcico apresentaram 5,45\% de nitrogênio, um valor aproximadamente $10 \%$ mais alto. Como as excretas de poedeiras são muito utilizadas como fertilizantes em diversas culturas, estudos agronômicos devem ser realizados para verificar o valor do nitrogênio como fertilizante, o que evita seu desperdício ou a contaminação ambiental.

O fosfato monoamônio resultou em consumo médio de ração aproximadamente $13 \%$ superior $(\mathrm{P}<0,05)$ em comparação ao obtido com o bicálcico (Tabela 3). Os calcários não influenciaram esta variável, logo, a diferença de granulometria não afetou o consumo alimentar.

A conversão alimentar, avaliada pela massa de ovos, foi significativamente melhor $(\mathrm{P}<0,05)$ nas aves que receberam o fosfato monoamônio como fonte de fósforo. Apesar do maior consumo de ração obtido com o fosfato monoamônio, houve maior produção de massa de ovos, o que melhorou a eficiência alimentar. Este resultado pode ser explicado pela maior disponibilidade de fósforo desse fosfato, o que possibilita sua melhor utilização no metabolismo das aves.

Não houve efeito $(\mathrm{P}>0,05)$ dos fosfatos sobre o percentual e a espessura da casca dos ovos (Tabela 4). Esses resultados indicam que o calcário calcítico Paraíso é tão eficiente quanto o Barroso no suprimento de cálcio durante a formação da casca dos ovos. Mesmo com a solubilidade maior no início da digestão, proporcionada pela menor granulometria,

Tabela 3 - Desempenho zootécnico de poedeiras alimentados com rações contendo diversas fontes de cálcio e fósforo

\begin{tabular}{lllrr}
\hline Variável & \multicolumn{4}{c}{ Fonte de cálcio e fósforo } \\
\cline { 2 - 4 } & FB/CB & FB/CP & MAP/CB & MAP/CP \\
\hline Produção de ovos (\% ave-dia) & $66,40 \mathrm{~B}$ & $67,30 \mathrm{~B}$ & $75,40 \mathrm{~A}$ & $79,37 \mathrm{~A}$ \\
Peso médio dos ovos (g) & $59,96 \mathrm{~B}$ & $58,86 \mathrm{~B}$ & $61,97 \mathrm{~A}$ & 93,70 \\
Massa de ovos (g) & $39,71 \mathrm{~B}$ & $40,38 \mathrm{~B}$ & $46,82 \mathrm{~A}$ & $50,11 \mathrm{~A}$ \\
Consumo médio de ração (g) & $92,1 \mathrm{~B}$ & $93,5 \mathrm{~B}$ & 10,35 \\
Conversão alimentar (kg/kg ovo) & 2,377A & $2,373 \mathrm{~A}$ & $105,8 \mathrm{~A}$ & $104,4 \mathrm{~A}$ \\
\hline
\end{tabular}

FBC/CB - fosfato bicálcico e calcário calcítico Barroso (cinza); FBC/CP- fosfato bicálcico e calcário calcítico Paraíso (branco); MAP/CB - fosfato monoamônio e calcário calcítico Barroso; MAP/CP - fosfato monoamônio e calcário calcítico Paraíso.

Médias seguidas de letras diferentes $(P<0,05)$ nas linhas diferem pelo teste Tukey. 
Tabela 4 - Qualidade da casca dos ovos de poedeiras alimentadas com rações com diversas fontes de cálcio e fósforo

\begin{tabular}{|c|c|c|c|c|c|}
\hline Variável & \multicolumn{4}{|c|}{ Tratamento } & CV $(\%)$ \\
\hline Porcentagem da casca (\%) & 9,81 & 9,86 & 9,79 & 9,70 & 4,77 \\
\hline
\end{tabular}

FBC/CB - fosfato bicálcico e calcário calcítico Barroso (cinza); FBC/CP- fosfato bicálcico e calcário calcítico Paraíso (branco); MAP/CB - fosfato monoamônio e calcário calcítico Barroso; MAP/CP - fosfato monoamônio e calcário calcítico Paraíso.

Médias seguidas de letras diferentes nas linhas diferem $(P<0,05)$ pelo teste Tukey.

o calcário calcítico Paraíso não prejudicou a resistência das cascas dos ovos.

Não foram observadas durante o período experimental fezes liquefeitas ou prejuízos na estrutura esquelética das aves que possam ser atribuídos ao teor de magnésio do calcário Paraíso (1,92\%). As taxas de viabilidade dos ovos das aves em produção foram 100; 97,5; 97,5 e 94,5\% FBC/CB; FBC/CP; MAP/CB e MAP/CP, respectivamente, o que não comprova qualquer efeito do maior nível de magnésio sobre a taxa de mortalidade das aves. A utilização de 12\% de calcário calcítico Paraíso nas rações experimentais, aproximadamente $0,23 \% \mathrm{Mg}$ (2300 ppm), não foi suficiente para provocar toxidez nas poedeiras. Esse nível é muito inferior às concentrações de 11.200 a 19.600 ppm de Mg descritas pelo NRC (1994) como níveis dietéticos tóxicos capazes de reduzir a produção de ovos.

\section{Conclusões}

O fosfato monoamônio (MAP) pode ser utilizado como fonte alternativa de fósforo em substituição ao fosfato bicálcico. O calcário Paraíso é tecnicamente viável para utilização na alimentação de poedeiras comerciais, pois não prejudica a produção e a qualidade da casca dos ovos.

\section{Literatura Citada}

AXE, D. Solubility should be used in selection of limestone product. Feedstuffs, v.61, n.53, p.16-20, 1989.

BRONNER, F. Nutrient bioavailability with a special reference to calcium. Journal Nutrition, v.123, n.5, p.797-802, 1993.

BUTOLO, J.E. Qualidade de ingredientes na alimentação animal. Campinas: Agro Comunicação, 2002. 430p.

FASSANI, É.J.; BETERCHINI, A.G.; KATO, R.K. et al. Composição e solubilidade in vitro de calcários calcíticos de Minas Gerais. Ciências Agrotécnica, v.28, n.4, p.913-918, 2004.

HM MINÉRIOS INDUSTRIAIS. Características de calcário. Campina Grande: 1999. 5p. (Informativo Industrial, 1).

NATIONAL RESEARCH COUNCIL - NRC. Nutrient requirements of poultry. Washington, D.C.: National Academy Press, 1994. $155 p$.

PCS BRASIL. Informativo PCS fosfatos do Brasil. N.01/2003.

RAO, K.S.; ROLAND, D.A. Influence of dietary calcium level and particle size of calcium solubilization by commercial Leghorns. Poultry Science, v.68, n.11, p.1499-1505, 1989.

ROSTAGNO, H.S. Tabelas brasileiras para aves e suínos. Composição de alimentos e exigências nutricionais. 2.ed. Viçosa, MG: Editora UFV, 2005. 186p.

SUGAHARA, M. Fisiologia da postura e metabolismo de cálcio. Aves e Ovos, Agosto, p.18-22, 1993.

UNIVERSIDADE FEDERAL DE VIÇOSA - UFV. SAEG - Sistema de Análises Estatísticas e Genéticas. Viçosa, MG: 1999. (CD-ROM).

VELOSO, J.A.F. Perspectivas de uso dos fosfatos de rocha nacionais na alimentação animal. Caderno Técnico da Escola de Veterinária UFMG, v.6, p.55-84, 1991.

ZHANG, B.; COON, C.N. The relationship of calcium intake, source, size solubility in vitro and in vivo, and gizzard limestone retention in laying hens. Poultry Science, v.76, n.12, p.1702-1706, 1997. 